\title{
Avances en la cirugía de tumores del páncreas exocrino en niños
}

\author{
JUAN CARLOS PATTILLO S. ${ }^{1}$, NICOLÁS JARUFE C. ${ }^{2}$ \\ 1. Sección Cirugía Pediátrica, División de Cirugía, Facultad de Medicina, Pontificia Universidad Católica de Chile. \\ 2. Departamento de Cirugía Digestiva, División de Cirugía, Facultad de Medicina, Pontificia Universidad Católica de Chile.
}

\begin{abstract}
Advances in the surgical management of pancreatic exocrine tumors in children

Introduction: Pancreatic tumors in childhood are rare and diverse. Among them, pancreatoblastoma is the most common pancreatic tumor in young children, followed by the solid pseudopapillary tumor (SPT), which is an uncommon neoplasm characterized by low potential for malignancy that mainly occurs in young women. Complete surgical resection is the treatment of choice. Following the advances in minimally invasive surgery, laparoscopic distal pancreatectomy appears to be feasible and safe in children. Objective: To report a case of a child with SPT to create awareness on this topic and update its surgical management. Case report: The case of a 10 year old boy carrying a pancreatic tumor, who sought medical attention due to abdominal pain after minor trauma, is reported. Ultrasonography and CT scan of the abdomen showed an $8 \mathrm{~cm}$ tumor in the body and tail of the pancreas, no metastases. The alpha-fetoprotein was normal. Laparoscopic corporocaudal pancreatectomy and splenectomy were performed, and the patient was discharged on the sixth postoperative day without incident. Biopsy confirmed SPT. Discussion: This study exposes the characteristics and classification of pancreatic tumors in childhood. In particular, SPT is discussed regarding diagnosis and the advantages of minimally invasive surgery as part of the treatment.
\end{abstract}

(Key words: Tumor, pancreas, pancreatectomy, laparoscopic, children).

Rev Chil Pediatr 2013; 84 (4): 417-424

\section{RESUMEN}

Introducción: Los tumores pancreáticos en la infancia son infrecuentes y diversos. Dentro de ellos, el pancreatoblastoma es el más frecuente en niños pequeños, seguido por el tumor sólido pseudopapilar (TSP), que es una neoplasia de bajo grado de malignidad que predomina en niñas y mujeres jóvenes. La resección quirúrgica completa, es su tratamiento de elección. En la medida del progreso de la cirugía mínimamente invasiva, la pancreatectomía laparoscópica parece ser factible y segura en niños. Objetivo: Comunicar el caso clínico de un niño con TSP para alertar sobre esta patología y actualizar acerca de su manejo quirúrgico. Caso

Recibido el 3 de mayo de 2013, devuelto para corregir el 10 de junio de 2013, segunda versión 1 de julio de 2013, tercera versión 08 de agosto de 2013, aceptado para publicación el 12 de agosto de 2013.

Declaración: Ninguno de los autores tiene conflictos de interés ni ha existido financiamiento para este artículo. Esta investigación ha sido aprobada por el Comité de Ética en Investigación de la Pontificia Universidad Católica de Chile (proyecto 13-149).

Este trabajo cumple con los requisitos sobre consentimiento /asentimiento informado, comité de ética, financiamiento, estudios animales y sobre la ausencia de conflictos de intereses según corresponda.

Correspondencia a:

Juan Pattillo S.

E-mail: jpattill@med.puc.cl 
clínico: Niño de 10 años portador de un tumor pancreático diagnosticado por presentar dolor abdominal luego de un traumatismo menor. La ultrasonografía y TAC de abdomen mostraron un tumor de $8 \mathrm{~cm}$ en el cuerpo y cola del páncreas, sin metástasis. La a-feto proteína fue normal. Se realizó pancreatectomía córporo caudal laparoscópica y esplenectomía, siendo dado de alta al sexto día postoperatorio, sin incidentes. La biopsia confirmó TSP. Discusión: Se expone acerca de las características y clasificación de los tumores del páncreas en la infancia. En particular de discute acerca del TSP, su enfrentamiento diagnóstico y las ventajas de la cirugía mínimamente invasiva en su tratamiento.

(Palabras clave: Tumor páncreas, pancreatectomía, laparoscopía, niños).

Rev Chil Pediatr 2013; 84 (4): 417-424

\section{Introducción}

Los tumores pancreáticos en la infancia son infrecuentes y heterogéneos ${ }^{1}$. De estos, el pancreatoblastoma $(\mathrm{PB})$ es el tumor más frecuente en niños pequeños ${ }^{2}$. El tumor sólido pseudopapilar (TSP) es considerado una neoplasia de bajo grado de malignidad, se presenta predominantemente en niñas adolescentes y mujeres jóvenes, y la resección quirúrgica completa es su tratamiento de elección ${ }^{3}$. Las formas de manifestación clínica más frecuente del TSP son el dolor abdominal inespecífico y la presencia de masa abdominal. También pueden ser descubiertos en forma incidental en pacientes asintomáticos. En la medida que las técnicas de cirugía mínimamente invansivas han evolucionado, la pancreatectomía laparoscópica parece ser factible y segura en niños ${ }^{4}$.

El objetivo de nuestro trabajo es comunicar el caso clínico de un niño con TSP para alertar sobre esta patología y actualizar acerca de su manejo quirúrgico.

\section{Caso clínico}

Niño de 10 años de edad, que consultó en un servicio de urgencia por dolor abdominal persistente y vómitos. El paciente refirió haber recibido un golpe de moderada intensidad mientras jugaba un partido de fútbol, dos días antes de consultar. Posteriormente, presentó dolor abdominal epigástrico mal caracterizado, intenso, asociado a vómitos alimentarios en seis oportunidades y alza térmica autolimitada hasta $37,7^{\circ} \mathrm{C}$. Al momento de su primera atención, el paciente se encontraba quejum- broso pero en buenas condiciones, afebril y sin hallazgos al examen físico. Debido al antecedente del trauma y al dolor persistente se realizó una ecografía abdominal que mostró un tumor heterogéneo del páncreas, por lo que fue derivado a nuestro centro.

A su ingreso el paciente se encontraba asintomático, afebril, hemodinámicamente estable. El examen físico era normal, destacando un abdomen blando, depresible e indoloro, sin signos de irritación peritoneal y sin masa palpable. Se completó el estudio con tomografía axial computada (TAC) abdominal que mostró un extenso tumor localizado en el cuerpo y cola del páncreas, de $8 \mathrm{~cm}$ de diámetro mayor, encapsulado y con algunas áreas hipodensas en su interior, sin calcificaciones. El tumor desplazaba la vena y la arteria esplénica, comprimiéndolas, pero sin evidencia de compromiso vascular ni de metástasis (figuras 1a y 1b). Se planteó como diagnósticos diferenciales PB y TSP. Del resto de sus exámenes de laboratorio destacaban niveles normales de lipasa (81 UI/1, valor normal 23-300 UI/1) y de $\alpha$-feto proteína $(1.3 \mathrm{ng} / \mathrm{ml}$, valor normal $<8,1 \mathrm{ng} / \mathrm{l})$. Se programó una pancreatectomía córporo-caudal por vía laparoscópica.

La cirugía se inició estableciendo un neumoperitoneo de $12 \mathrm{mmHg}$ con aguja de Verres e insertando un trócar umbilical de $12 \mathrm{~mm}$. Se utilizó un segundo trócar de $12 \mathrm{~mm}$ en el flanco izquierdo y dos trócares auxiliares de $5 \mathrm{~mm}$ en ambos flancos. Un quinto trócar de $5 \mathrm{~mm}$ se insertó en el epigastrio para retracción del estómago.

La cirugía se realizó con disector ultrasónico y gancho monopolar, accediendo a la transcavidad y respetando los vasos gastroepiploí- 


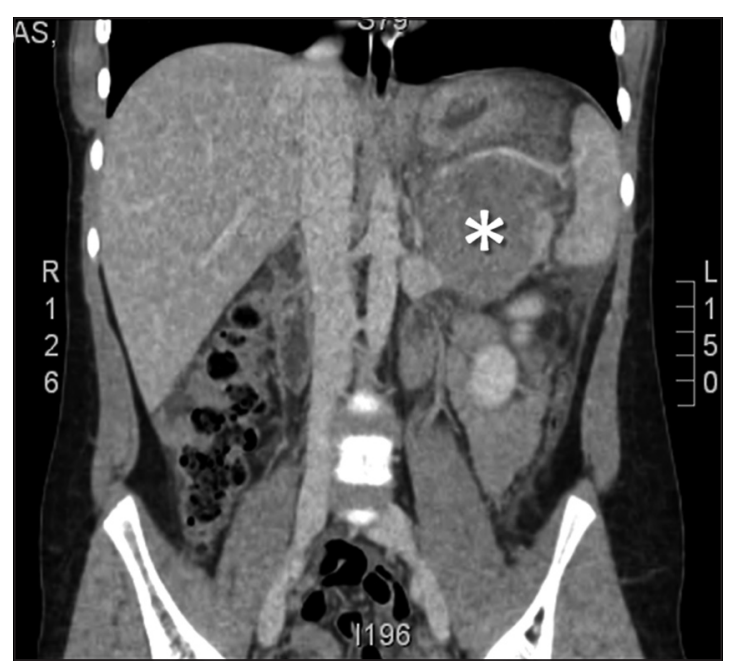

Figura 1a. TAC abdominal con contraste, corte coronal. Se observa una lesión tumoral en la cola del páncreas (*). Nótese la compresión y desplazamiento hacia cefálico de los vasos esplénicos, visibles sobre el tumor.

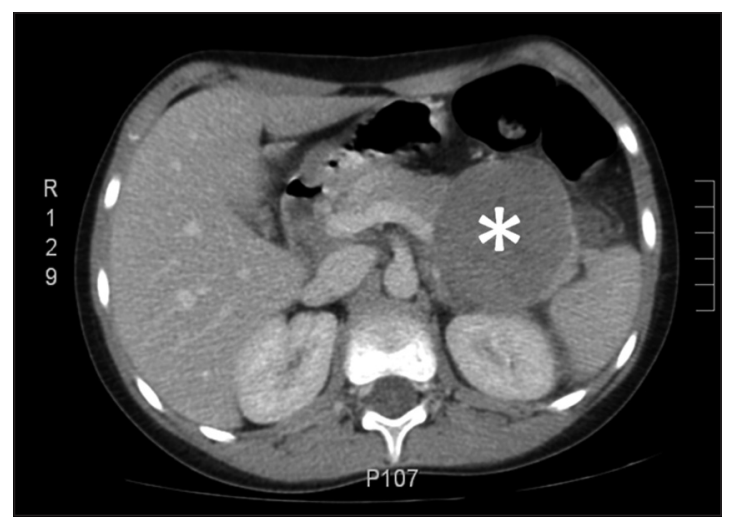

Figura 1b. TAC abdominal con contraste, corte transversal. Se visualiza un tumor heterogéneo, con áreas hipodensas, bien encapsulado, en la cola del páncreas.

cos. Se movilizó completamente el estómago exponiendo el tumor (figura 2a). Se liberó el borde inferior del páncreas identificando las venas mesentéricas superior e inferior, la vena esplénica y la vena porta. Se completó la disección sobre el páncreas identificando el tronco celíaco y la arteria esplénica, con esto se logró pasar detrás del páncreas para proceder a la sección corporal con stapler vascular de $65 \mathrm{~mm}$ (figura 2b). Se seccionaron los vasos esplénicos luego de obtener control vascular con Hem-o-lock, continuando con la disección

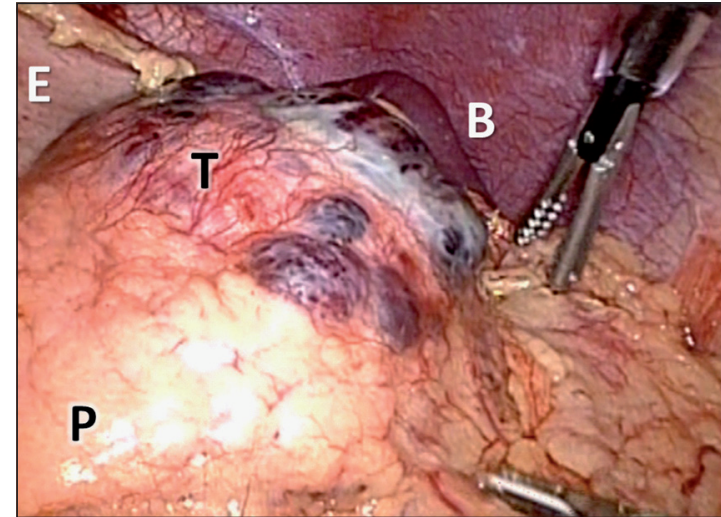

Figura 2a. Captura de imagen de vídeo, cirugía laparoscópica. El omento se ha abierto, accediendo a la transcavidad, el estómago ha sido desplazado hacia cefálico, siendo visible su cara posterior (E). Se observa el páncreas $(\mathrm{P})$ ocupado en su extremo distal por una lesión encapsulada, de aspecto hemorrágico (T). Al fondo de la imagen es visible el bazo (B). El hilio esplénico se encontraba en íntima relación con la lesión tumoral.

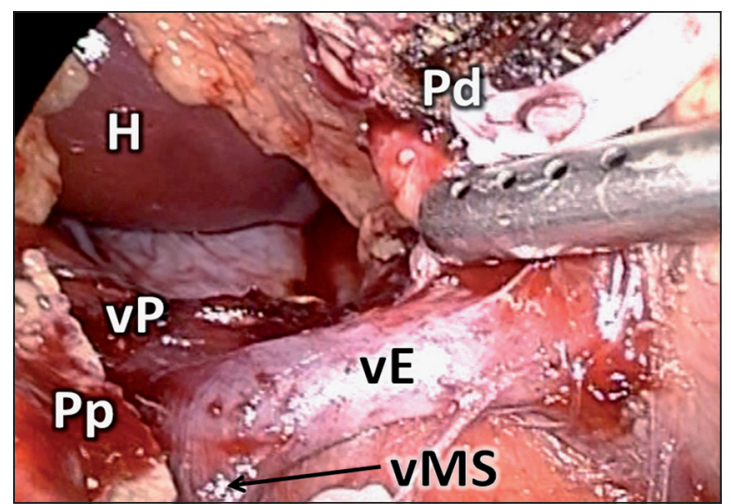

Figura 2b. Captura de imagen de vídeo, cirugía laparoscópica. El estómago se ha levantado y se ha seccionado el páncreas, se observan las líneas de sección proximal (Pp) y el borde de sección distal del páncreas (Pd). Se observan las venas esplénica (vE) y mesentérica superior (vMS) en su confluencia para formar la vena porta (vP). Al fondo el lóbulo caudado del hígado $(\mathrm{H})$.

hacia distal, donde se comprobó que el tumor comprometía íntimamente el hilio esplénico. Se decidió incluir el bazo en la resección, sin entrar en el hilio esplénico, para asegurar una resección completa. Durante el procedimiento hubo una ruptura de la cápsula del tumor en relación a la introducción de un instrumento fuera del campo de visión. Se extrajo un trozo de cápsula por separado sin identificar tumor 
macroscópico remanente. La pieza se introdujo en una bolsa y se extrajo a través de una incisión Pfannenstiel. Se realizó aseo, revisión de hemostasia y se dejó un drenaje al lecho pancreático cercano al borde de sección.

El paciente tuvo una evolución postoperatoria sin incidentes, iniciando alimentación por vía oral a las 48 h de la cirugía. El drenaje se mantuvo por cuatro días con escasa secreción de líquido serohemático, comprobándose niveles normales de amilasa (65 U/L) previo a su retiro. El niño fue dado de alta al sexto día post operatorio.

El análisis de la biopsia diferida informó un TSP del páncreas, con bordes quirúrgicos negativos, sin permeaciones vasculares ni compromiso de ganglios linfáticos regionales. El estudio inmunohistoquímico fue positivo para beta-catenina (patrón de tinción nuclear y citoplasmático en células tumorales) y CD10, positivo para CD56 en algunas células tumorales y negativo para cromogranina y sinaptofisina.

Durante el seguimiento clínico a 4 meses el paciente se encuentra asintomático y presenta un buen resultado cosmético (figura 3 ).

\section{Discusión}

Los tumores del páncreas en niños pueden clasificarse según su origen en epiteliales y no epiteliales, siendo estos últimos extremadamente infrecuentes. Los tumores epiteliales pueden clasificarse a su vez en tumores exocrinos y endocrinos (tabla 1). Dentro de los tumores exocrinos del páncreas, el $\mathrm{PB}$ es el más frecuente en niños pequeños, ocurriendo preferentemente durante la primera década de la vida ${ }^{2}$. Ha habido casos descritos en fetos, asociados al síndrome de Beckwith-Wiedemann $^{5,6}$ y unos pocos casos en adultos. En las imágenes, el PB es un tumor heterogéneo, muchas veces multiloculado, con tabicaciones hiperecogénicas que se realzan con el uso de contraste.

El TSP es más frecuente en niñas adolescentes y mujeres jóvenes, son tumores incidentales y su presencia en pacientes de sexo masculino es excepcional. También presenta una arquitectura interna heterogénea, con áreas sólidas y quísticas, con hemorragia y necrosis.

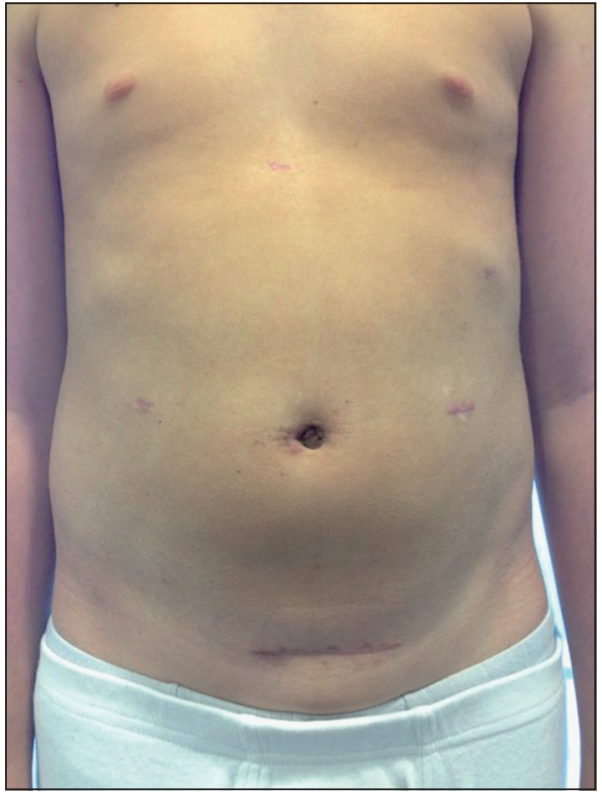

Figura 3. Fotografía postoperatoria, el resultado cosmético favorable es evidente, apreciándose incisiones pequeñas para la inserción de trócares. La incisión suprapúbica se utilizó para extraer la pieza y queda cubierta con la ropa interior.

Un rasgo particular del TSP es la presencia de una cápsula fibrosa y de elementos hemorrágicos. El diagnóstico por imágenes resulta fundamental a la hora de decidir la vía de abordaje y la factibilidad de una resección quirúrgica ${ }^{7}$. La presentación clínica más frecuente es el dolor abdominal, seguido de masa abdominal palpable. El dolor abdominal es inespecífico y de difícil caracterización, muchas veces puede tratarse de un cuadro crónico de varios meses de evolución. Dado que el páncreas es un órgano retroperitoneal, ubicado bajo el reborde costal, para que un tumor pancreático sea palpable debe alcanzar dimensiones considerables $^{8}$. Otras formas menos frecuentes de presentación son la ruptura tumoral, habitualmente secundaria a trauma, y la pancreatitis aguda. Por esto, en pacientes con pancreatitis aguda, aunque sea leve, siempre debiera realizarse un estudio con imágenes. El TSP puede asociarse a síntomas como saciedad precoz, sensación de plenitud e incluso reflujo gastroesofágico, considerados a veces como funcionales o banales, lo que puede retardar el estudio diagnós- 
Tabla 1. Clasificación de los tumores pancreáticos de la infancia

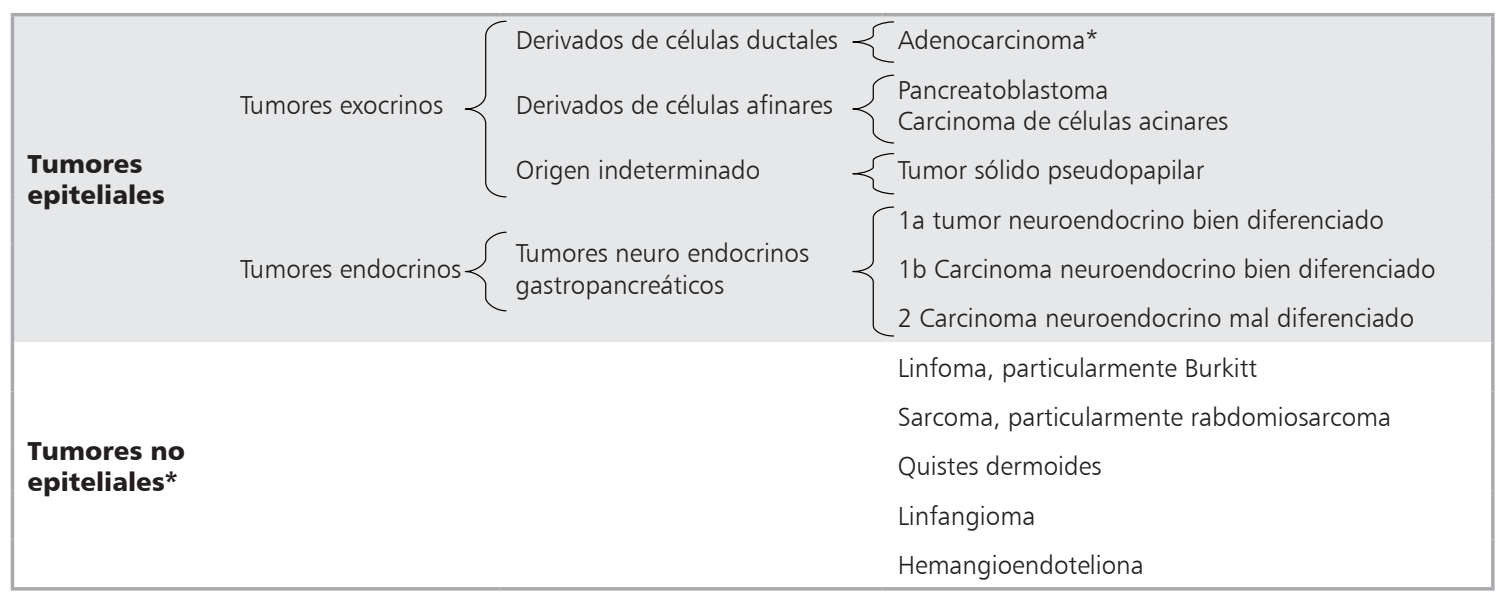

tico con imágenes ${ }^{9}$. En la gran mayoría de los niños el diagnóstico de tumor pancreático se establece con una ecografía abdominal realizada en el contexto de un paciente con dolor abdominal inespecífico (tabla 2).

Estos tumores pueden ser bien caracterizados con TAC o resonancia magnética, las que contribuyen a diferenciarlos de lesiones inflamatorias del páncreas, como pancreatitis aguda o pseudoquistes. Ambos exámenes permiten tener una idea de la ubicación y tamaño de la lesión, estructura interna, su relación con los elementos vasculares y la presencia de compromiso regional o a distancia ${ }^{10}$. Al momento de su presentación, el $85 \%$ de los TSP se limitan al páncreas ${ }^{11}$.

Al estudio histológico son tumores marcadamente homogéneos, con estructuras sólidas, pseudopapilares y quísticas en distintas proporciones. Esto último significó que, desde la primera descripción de este tumor realizada por Franz en $1959^{12}$, haya recibido múltiples denominaciones según el patrón histológico predominante. En $1981 \mathrm{Kloppe}^{13}$, distingue al TSP como entidad clínica definida y posteriormente la OMS los incluye dentro de su clasificación de tumores del páncreas exocrino como "tumor sólido pseudopapilar", siendo ésta la denominación utilizada en la actualidad ${ }^{14}$.

En el estudio inmunohistoquímico, las células neoplásicas son difusamente positivas para vimentina, la mayoría también lo son para enolasa neuronal específica y sólo algunas po-
Tabla 2. Hallazgos clínicos en 643 pacientes con TSP

\begin{tabular}{|lc|}
\hline Hallazgos clínicos & $\%$ \\
\hline Dolor abdominal & 46,50 \\
\hline Masa abdominal & 34,84 \\
\hline Asintomático & 15,55 \\
\hline Disconfort / dispepsia & 3,89 \\
\hline Vómitos & 3,89 \\
\hline Náuseas & 3,27 \\
\hline Antecedente de trauma & 3,11 \\
\hline Fiebre & 1,24 \\
\hline Ictericia & 1,09 \\
\hline Baja de peso & 0,93 \\
\hline Otros síntomas & 0,93 \\
\hline Anorexia & 0,78 \\
\hline Pseudoquiste pancreático & 0,62 \\
\hline
\end{tabular}

(Adaptado de Papavramidis and Papavramidis, "Solid pseudopapillary tumors of the pancreas: review of 718 patients reported in English literature" J Am Coll Surg. 2005; 200(6): 965-72.

cas células, y en forma focal, son positivas para citoqueratinas, synaptofisina y S-100. El patrón citoplasmático y nuclear de beta catenina está presente en prácticamentes todas las células del tumor en la gran mayoría de los casos. La expresión de CD56 y CD10 y de otros marcadores neuroendocrinos en forma local es única para los TSP por lo que resultan útiles al momento de establecer el diagnóstico. Estos hallazgos sugieren que los TSP se originan de células que han desarrollado características 
exocrinas, pero que tienen capacidad de diferenciación dual ${ }^{15}$.

A diferencia de los tumores exocrinos, los tumores endocrinos suelen tener manifestaciones hormonales tempranas y se distinguen por su tamaño pequeño, apariencia homogénea e intenso realce con el contraste endovenoso en los estudios de imágenes. Estos tumores derivan de células del sistema neuroendocrino (APUD $)^{16}$, diseminadas a través de la mucosa del tracto gastrointestinal o formando grupos celulares como los islotes de Langerhans en el páncreas, y se denominan tumores neuroendocrinos gastropancreáticos o GEP-NET por sus siglas en inglés ${ }^{17}$.

El estudio inicial de niños con sospecha de tumores pancreáticos incluye la caracterización del tumor primario y la evaluación de metástasis a distancia. Al enfrentarnos con un tumor encapsulado, bien localizado y sin evidencia de metástasis la primera aproximación debiera ser un intento de resección quirúrgica curativa. Las terapias adyuvantes o neoadyuvantes, incluyendo manejo hormonal, quimioterapia y radioterapia, debieran reservarse para casos en los que la cirugía curativa no es posible. La evidencia muestra que la eficacia de estos tratamientos es anecdótica, debiendo ajustarse los esquemas según el tipo histológico del tumor y su estadio. En estos casos se recomienda la realización de biopsia del tumor primario $^{8}$ (figura 4).

Con el avance de las técnicas de cirugía mínimamente invasivas, la resección de tumores pancreáticos benignos o de bajo grado de malignidad por vía laparoscópica ha demostrado ser una técnica factible y que ofrece ventajas cosméticas evidentes, facilita el manejo del dolor perioperatorio y tiene un menor impacto fisiológico en el paciente ${ }^{18-20}$. El primer caso de pancreatectomía laparoscópica en un paciente pediátrico por TSP fue publicado en $2003^{4}$. Desde entonces y hasta la fecha es posible encontrar al menos 8 pacientes pediátricos con TSP sometidos a este procedimiento que han sido comunicados en la literatura de habla inglesa ${ }^{19,21-24}$. Hemos publicado recientemente nuestra experiencia en cirugía del $\mathrm{TSP}^{25}$, donde 7 de 11 pacientes fueron manejados por vía laparoscópica (6 pancreatectomías córporocaudales y una pancreatoduodenectomía completamente laparoscópica), hubo una conversión a cirugía abierta y se incluyó a una paciente pediátrica de 14 años. Dicha paciente fue dada de alta al $5^{\circ}$ día postoperatorio; una semana después de la cirugía se evidenció una fístula pancreática que requirió un aseo quirúrgico, el que fue realizado por vía laparoscópica, permaneciendo hospitalizada por 6 días más. La formación de fístulas pancreáticas es

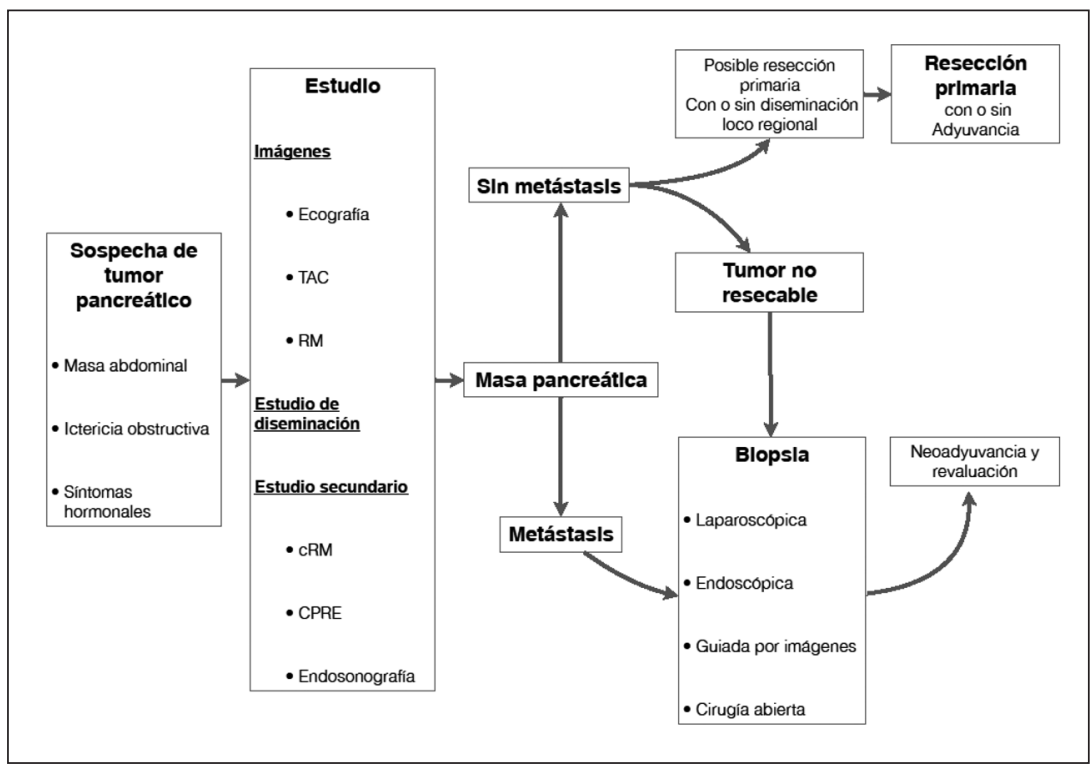

Figura 4. Algoritmo manejo tumores pancreáticos en niños (Modificado de Yu, ref. 8). 
una complicación frecuente en las pancreatectomías, tanto abiertas como laparoscópicas (5 a $30 \%)^{22,26}$, y su manejo puede ser facilitado al utilizar drenajes en forma rutinaria ${ }^{25}$.

La resección quirúrgica radical es el tratamiento de elección del TSP. La enucleación o la resección parcial de estos tumores, que ha sido apoyada por algunos autores ${ }^{3}$, se ha asociado a recidiva y mortalidad en algunos casos de pacientes pediátricos ${ }^{27,28}$.

Se han descrito recidivas tumorales y la aparición de mestástasis hasta 10 años después de una cirugía ${ }^{29}$. Si bien no se ha podido determinar en forma estadísticamente significativa los factores de riesgo que pudieran predecir una recidiva tumoral, existen ciertos hallazgos descriptivos que indicarían una mayor posibilidad de presentarla: la presencia de metástasis en la cirugía inicial, invasión vascular o de órganos vecinos, gran tamaño tumoral (mayor de $13 \mathrm{~cm}$ ), paciente joven al momento de la presentación, ruptura tumoral, resección incom$\operatorname{pleta}^{29}$. La realización de biopsias, ya sea por percutáneas o por vía laparoscópica, pareciera ser un factor de riesgo en la aparición de recidivas tumorales, por lo que debieran evitarse en el caso de tumores factibles de resecar ${ }^{21,30}$. En nuestro paciente, la presencia de una ruptura tumoral asociada al procedimiento laparoscópico podría influir en el desarrollo de recidivas, por lo que hemos establecido un programa de seguimiento cercano, con ecografía abdominal y resonancia magnética de abdomen intercalados cada 6 meses. A pesar de la presencia de una recidiva tumoral, el pronóstico de los pacientes con TSP sigue siendo excelente con un buen seguimiento y tratamiento quirúrgico agresivo ${ }^{29}$.

El presente caso, junto con el publicado en la serie anterior ${ }^{25}$, son los primeros casos de cirugía laparoscópica de TSP en niños comunicados en nuestro país.

En conclusión, el TSP es un tumor infrecuente en niños. Su diagnóstico es difícil, muchas veces incidental. Por esto, es importante que en pacientes con síntomas abdominales persistentes, aunque sean inespecíficos, se plantee la realización de estudios de imágenes. La cirugía laparoscópica del TSP en niños es factible y segura. El seguimiento a largo plazo es fundamental al momento de determinar el rol de la laparoscopía en el manejo de estos tumores. La cirugía mínimamente invasiva de estos pacientes debiera ser realizada por equipos con experiencia en cirugía laparoscópica avanzada, particularmente en las áreas hepatobiliar y pancreática.

\section{Agradecimientos}

Los autores agradecen al Dr. Paul Harris, División de Pediatría, Facultad de Medicina, Pontificia Universidad Católica de Chile, por la revisión crítica y sugerencias realizadas al manuscrito.

\section{Referencias}

1.- Jaksic T, Yaman M, Thorner P, et al: A 20-year review of pediatric pancreatic tumors. J Pediatr Surg 1992; 27 (10): 1315-7.

2.- Solcia E, Capella C, Kloppel G: Atlas of tumor pathology: tumors of the pancreas. Washington, DC: Armed Forces Institute of Pathology, 1997.

3.- Acuña R, Soto P, Muñoz V, Acuña J: Tumor sólido pseudopapilar de páncreas, reporte de un caso. Rev Chil Pediatr 2008; 79 (4): 404-8.

4.- Carricaburu E, Enezian G, Bonnard A, et al: Laparoscopic distal pancreatectomy for Frantz's tumor in a child. Surg Endosc 2003; 17 (12): 2028-31.

5.- Pelizzo G, Conoscenti G, Kalache KD, et al: Antenatal manifestation of congenital pancreatoblastoma in a fetus with Beckwith-Wiedemann syndrome. Prenat Diagn 2003; 23 (4): 292-4.

6.- Drut R, Jones MC: Congenital pancreatoblastoma in Beckwith-Wiedemann syndrome: an emerging association. Pediatr Pathol 1988; 8 (3): 331-9.

7.- Chung E, Travis M, Conran R: Pancreatic Tumors in Children: Radiologic-Pathologic Correlation. Radiographics 2006; 26: 1211-38.

8.- Yu D, Kozakewich H, Pérez-Atayde A, et al: Childhood pancreatic tumors: a single institution experience. J Peditr Surg 2009; 44: 2267-72.

9.- Papavramidis T, Papavramidis S: Solid pseudopapillary tumors of the pancreas: review of 718 patients reported in English literature. J Am Coll Surg 2005; 200 (6): 96572.

10.- $H u$ S, Lin $X$, Song $Q$, Chen $K$ : Solid pseudopapillary tumour of the pancreas in children: clinical and compu- 
ted tomography manifestation. Radiol Med 2012; 117 : 1242-9.

11.- Lack EE, Cassady JR, Levey R, Vawter GF: Tumors of the exocrine pancreas in children and adolescents. A clinical and pathologic study of eight cases. Am J Surg Pathol 1983; 7: 319-27.

12.- Frantz VK: Tumors of the pancreas. In: Atlas of tumor pathology, section VII, fascicles 27 and 28. Washington, DC: Armed Forces Institute of Pathology; 1959.

13.- Kloppel G, MorohoshiT, John HD, et al: Solid and cystic acinar cell tumour of the pancreas. Virch Arch A Pathol Anat 1981; 392: 171-83.

14.- Kloppel G, Solcia E, Longnecker DS, et al: Histological typing of tumors of the exocrine pancreas. 2nd ed. (WHO international histological classification of tumors). Berlin, Heidelberg,New York: Springer; 1996.

15.- Notohara K, Hamazaki S, Tsukayama C, et al: Solidpseudopapillary tumor of the pancreas. Immunohistochemical localization of neuroendocrine markers and CD10. Am J Surg Pathol 2000; 24: 1361-71.

16.- Klöppel G; Heitz PU: Die disseminierten (diffusen) endokrinen Zellen. In Spezielle pathologische Anatomie, Bd 14. W. Doerr \& G. Seifert, Eds.: 1079-135. Springer. Berlin. 1981.

17.- Klöppel G, Perren A, Heitz PU: The gastroenteropancreatic neuroendocrine cell system and its tumors: the WHO classification. Ann N Y Acad Sci 2004; 1014: 13-27.

18.- Mabrut JY, Fernández-Cruz L, Azagra JS, et al: Laparoscopic pancreatic resection: results of a multicenter European study of 127 patients. Surgery 2005; 137 : 597-605.

19.- Tsai F, Lee J, Chang Y: Laparoscopic Resection of a Giant Solid Pseudopapillary Neoplasm of Uncinate Process of the Pancreas in a Child. J Laparoendosc Adv Surg Tech 2011; 21 (19): 979-82

20.- Alvise C, Giovanni B, Despoina D, et al: Laparoscopic pancreatectomy for solid pseudo-papillary tumors of the pancreas is a suitable technique; our experience with long-term follow-up and review of the literature. Ann Surg Oncol 2011; 18: 352-7.

21.- Fais P, Carricaburu E, Sarnacki $S$, et al: Is laparoscopic management suitable for solid pseudo-papillary tumors of the pancreas? Pediatr Surg Int 2009; 25: 617-21.

22.- Melotti G, Cavallini A, Butturini G, et al: Laparoscopic distal pancreatectomy in children: Case report and review of the literature. Ann Surg Oncol 2007; 14: 1065 9.

23.- Uchida H, Goto C, Hiroshi Kishimoto H, et al: Laparoscopic spleen-preserving distal pancreatectomy for solid pseudopapillary tumor with conservation of splenic vessels in a child. J Peditr Surg 2010; 45: 1525-9.

24.- Sokolov YY, Stonogin SV, Donskoy DV, Povarnin OY, Vilesov AV: Laparoscopic pancreatic resections for solid pseudopapillary tumor in children. Eur J Pediatr Surg 2009; 19: 399-401.

25.- Slako M, Muñoz C, Briceño E, et al: Tumor sólido pseudopapilar del páncreas: estudio descriptivo de una serie de casos. Rev Chilena de Cirugía 2012; 64 (3): 251-6.

26.- Park AE, Heniford BT: Therapeutic laparoscopy of the pancreas. Ann Surg 2002; 236: 149-58.

27.- Papavramidis T, Papavramidis $S$ : Solid pseudopapillary tumors of the pancreas: review of 718 patients reported in English literature. J Am Coll Surg 2005; 200 (6): 965 72.

28.- Zhou H, Cheng W, Lam KY, Chan GC, Khong PL, Tam $P K$ : Solid-cystic papillary tumor of the pancreas in children. Pediatr Surg Int 2001; 17 (8): 614-20.

29.- Kim C, Han D, Kim J, et al: Solid pseudopapillary tumor of the pancreas: Can malignancy be predicted? Surgery 2011; 149 (5): 625-34.

30.- Levy P, Auber A, Ruszniewski P: Do not biopsy solid psdudopapillary tumors of the pancreas. Endoscopy 2008; 40: 959 . 